\title{
Bounds for the zeros of unilateral octonionic polynomials
}

\author{
Rogério Serôdio, P. D. Beites and José Vitória
}

\begin{abstract}
In the present work it is proved that the zeros of a unilateral octonionic polynomial belong to the conjugacy classes of the latent roots of an appropriate lambda-matrix. This allows the use of matricial norms, and matrix norms in particular, to obtain upper and lower bounds for the zeros of unilateral octonionic polynomials. Some results valid for complex and/or matrix polynomials are extended to octonionic polynomials.
\end{abstract}

\section{Introduction}

One of the relevant aspects related to polynomials is their zeros, a classical subject named polynomial root-finding. In fact, the theory of polynomials over commutative fields and, in general, over commutative integral domains is well known.

Since familiar properties from the commutative setting do not hold in the noncommutative context, polynomials with coefficients in a non-commutative algebra or in a non-commutative and non-associative algebra remains of interest to many researchers.

In [25] and references therein the characterization of the zeros of quaternionic polynomials was studied, based on Niven's algorithm. Other numerical methods based on Newton, Weierstrass and based on Sebastião e Silva's methods have been given in $[15,16,28]$.

Key Words: Octonions, octonionic polynomials, matricial norms, bounds for the zeros. 2010 Mathematics Subject Classification: Primary 11R52; Secondary 12D10, 15A60.

Received: 06.04.2021

Accepted: 30.04 .2021 
The first mention of octonionic polynomials seems to be in [14], where Eilenberg and Niven asserted that their proof of the Fundamental Theorem of Algebra for quaternionic polynomials could be extended to octonionic polynomials.

A proof of the Fundamental Theorem of Algebra for octonionic polynomials was presented in [20] by Jou, who used a topological method similar to that in [14]. In the former reference, it was proved that an octonionic polynomial has at least one zero if there is a unique monomial of the highest degree.

A geometric description of the set of zeros of an octonionic polynomial was given by Datta and Nag in [8]. Moreover, they proved a generalization of the known De Moivre's formula for complex numbers, obtaining one for octonions which gives the zero set of the octonionic polynomial $x^{m}-\boldsymbol{o}$. Independently, Leite and Vitória arrived at the same result in [21].

More recently, Serôdio [26] proved that, despite belonging to the same set of conjugacy classes, the zeros of a non-monic octonionic polynomial do not coincide, in general, with the zeros of the corresponding monic polynomial. In [27] he searched for methods to construct octonionic polynomials with a prescribed set of zeros.

Although there are methods $[4,6,17,31]$ for solving polynomial equations over quaternion and octonion algebras, taking into account that it can be hard to compute the zeros of a polynomial, localization and bounds for them are very often valuable mathematical objects. In fact, they are useful when one only needs approximate values or starting values for algorithms. As can be seen in [1] and references therein, where left and right eigenvalues of quaternionic matrices were considered, the aspects of location and bounds have received attention in the quaternionic context.

Applying strategies whose main advantage is the use of real matrices, hence avoiding the octonionic eigenvalue problem, localization and bounds for the zeros of a unilateral octonionic polynomial are dealt with in this paper. In fact, we exploit a relation between a unilateral octonionic polynomial (and its roots) and a suitable real matrix (and its spectrum). More concretely, we apply a general principle for which, if a result on the spectrum of a particular family of matrices holds, then it is possible to establish, without effort, a result on the roots of polynomials with coefficients in some algebra.

For completeness, in Section 2, we recall some definitions and results which are needed in the following sections. In Section 3, upper bounds for norms of the zeros of a unilateral octonionic polynomial are obtained through upper bounds for norms of the latent roots of certain lambda-matrices. In particular, we generalize known results on complex polynomials, obtained by Cauchy [5], Dehmer [9], Deutsch [11], Marden [22], Melman [23], and Vitória [33], to unilateral octonionic polynomials. Moreover, lower bounds for norms of the 
zeros of a unilateral octonionic polynomial are also presented.

\section{Preliminaries}

In this section we recall definitions and results related to: the octonion algebra, unilateral octonionic polynomials, matricial norms and spectral radius, and matrix polynomials.

\subsection{Octonion Algebra}

Let

$$
\mathbb{O}=\left\{\sum_{\ell=0}^{7} o_{\ell} \boldsymbol{e}_{\ell}: o_{\ell} \in \mathbb{R}, \ell=0, \ldots, 7\right\}
$$

be the octonion semifield (also called non-associative division ring or nonassociative skew-field), where the addition is accomplished by adding corresponding coefficients and the multiplication table can be summarized by the relations

$$
e_{i} e_{j}=-\delta_{i j} e_{0}+\varepsilon_{i j k} e_{k}
$$

where $\delta_{i j}$ is the Kronecker delta, $\varepsilon_{i j k}$ is a Levi-Civita symbol, i.e., a completely antisymmetric tensor with a positive value +1 when $i j k=123,145,167,246$, $275,374,365$ and $\boldsymbol{e}_{\mathbf{0}}$ is the identity. This element will be omitted whenever it is clear from the context.

Given $\boldsymbol{o} \in \mathbb{O}$, it can be written as $\boldsymbol{o}=\operatorname{Re}(\boldsymbol{o})+\operatorname{Im}(\boldsymbol{o})$, where $\operatorname{Re}(\boldsymbol{o})=$ $o_{0}$ and $\operatorname{Im}(\boldsymbol{o})=\sum_{\ell=1}^{7} o_{\ell} \boldsymbol{e}_{\ell}$ are called the real part and the imaginary part, respectively. The conjugate of $\boldsymbol{o}$ is defined as $\overline{\boldsymbol{o}}=\operatorname{Re}(\boldsymbol{o})-\operatorname{Im}(\boldsymbol{o})$. The norm (in the sense of composition algebras, [3]) of $\boldsymbol{o}$, denoted by $n_{\boldsymbol{o}}$, and the trace of $\boldsymbol{o}$, denoted by $t_{\boldsymbol{o}}$, are given, respectively, by $n_{\boldsymbol{o}}=\overline{\boldsymbol{o}} \boldsymbol{o}=\boldsymbol{o} \overline{\boldsymbol{O}}=\sum_{\ell=0}^{7} o_{\ell}^{2}$ and $t_{\boldsymbol{o}}=2 \operatorname{Re}(\boldsymbol{o})=\boldsymbol{o}+\overline{\boldsymbol{o}}$. The inverse of a non-zero octonion $\boldsymbol{o}$ is $\boldsymbol{o}^{-1}=n_{\boldsymbol{o}}^{-1} \overline{\boldsymbol{o}}$.

The elements of the basis of $\mathbb{O}$ can also be written as

$$
\begin{aligned}
& e_{0}=1, \quad e_{1}=i, \quad e_{2}=j, \quad e_{3}=i j, \\
& e_{4}=k, \quad e_{5}=i k, \quad e_{6}=j k, \quad e_{7}=i j k .
\end{aligned}
$$

The octonions satisfy some properties. The most often used are presented in the following theorem.

Theorem 2.1 (Ward, [35]). Let $\boldsymbol{o}_{1}, \boldsymbol{o}_{2}, \boldsymbol{o}_{3} \in \mathbb{O}$. Then

(i) $n_{\boldsymbol{o}_{1}}=0$ if and only if $\boldsymbol{o}_{1}=0$, 
(ii) $n_{\boldsymbol{O}_{1} \boldsymbol{o}_{2}}=n_{\boldsymbol{O}_{2} \boldsymbol{o}_{1}}=n_{\boldsymbol{O}_{1}} n_{\boldsymbol{O}_{2}}$,

(iii) $\boldsymbol{o}_{1}=\sqrt{n_{\boldsymbol{o}_{1}}} \boldsymbol{u}$ for some unit octonion $\boldsymbol{u}$,

(iv) $\overline{\boldsymbol{O}_{1} \boldsymbol{O}_{2}}=\overline{\boldsymbol{O}_{2}} \overline{\boldsymbol{O}_{1}}$,

(v) $\overline{\boldsymbol{o}_{1}+\boldsymbol{o}_{2}}=\overline{\boldsymbol{o}_{1}}+\overline{\boldsymbol{o}_{2}}$,

(vi) $\overline{\boldsymbol{o}_{1}}=\boldsymbol{o}_{1}$ if and only if $\boldsymbol{o}_{1} \in \mathbb{R}$,

(vii) $\overline{\boldsymbol{o}_{1}^{-1}}=\left(\overline{\boldsymbol{o}_{1}}\right)^{-1}$,

(viii) $\left(\boldsymbol{o}_{1} \boldsymbol{o}_{2}\right)^{-1}=\boldsymbol{o}_{2}^{-1} \boldsymbol{o}_{1}^{-1}$.

In addition to these properties, it is well known that any two elements of O (an alternative algebra) generate an associative subalgebra, [17].

Due to the non-associativity, the octonion algebra cannot be isomorphic to the $8 \times 8$ real matrix algebra with the usual multiplication. Despite this, it is possible to consider a representation of the vector space automorphism of $\mathbb{O}$ induced by $x \mapsto A x$ where $A$ is the coordinate matrix of the left multiplication by the octonion "represented" by $A$.

For theoretical and computational reasons, we introduce a pseudo, in the sense of the previous paragraph, matrix representation of an octonion. For further pseudo representations of octonions see Tian [31]. In what follows, for simplicity but in the sense of the mentioned paragraph, we use the terms representation and representing without the adjective pseudo.

Definition 2.2 (Tian, [31]). The left matrix representation $\omega(\mathbf{o})$ of the octonion

$$
\mathbf{o}=\sum_{\ell=0}^{7} o_{\ell} e_{\ell}
$$

is

$$
\omega(\mathbf{o})=\left[\begin{array}{cccccccc}
o_{0} & -o_{1} & -o_{2} & -o_{3} & -o_{4} & -o_{5} & -o_{6} & -o_{7} \\
o_{1} & o_{0} & -o_{3} & o_{2} & -o_{5} & o_{4} & -o_{7} & o_{6} \\
o_{2} & o_{3} & o_{0} & -o_{1} & -o_{6} & o_{7} & o_{4} & -o_{5} \\
o_{3} & -o_{2} & o_{1} & o_{0} & o_{7} & o_{6} & -o_{5} & -o_{4} \\
o_{4} & o_{5} & o_{6} & -o_{7} & o_{0} & -o_{1} & -o_{2} & o_{3} \\
O_{5} & -o_{4} & -o_{7} & -o_{6} & o_{1} & o_{0} & o_{3} & o_{2} \\
o_{6} & o_{7} & -o_{4} & o_{5} & o_{2} & -o_{3} & o_{0} & -o_{1} \\
o_{7} & -o_{6} & o_{5} & o_{4} & -o_{3} & -o_{2} & o_{1} & o_{0}
\end{array}\right] .
$$


Associated with these matrices we have the usual subordinated matrix norms. Given $\boldsymbol{o}=\sum_{\ell=0}^{7} o_{\ell} \boldsymbol{e}_{\ell}$,

$$
\begin{aligned}
\|\boldsymbol{o}\|_{2} & \equiv\|\omega(\boldsymbol{o})\|_{2}=\sqrt{n_{\boldsymbol{o}}}=\sqrt{\sum_{\ell=0}^{7} o_{\ell}^{2}} \\
\|\boldsymbol{o}\|_{1} & \equiv\|\omega(\boldsymbol{o})\|_{1}=\sum_{\ell=0}^{7}\left|o_{\ell}\right| \\
\|\boldsymbol{o}\|_{\infty} & \equiv\|\omega(\boldsymbol{o})\|_{\infty}=\sum_{\ell=0}^{7}\left|o_{\ell}\right|
\end{aligned}
$$

Hence, $\|\boldsymbol{o}\|_{1}=\|\boldsymbol{o}\|_{\infty}$.

With the purpose of introducing a convenient matrix multiplication, we show another way of representing the octonions by a matrix which is closely related to the one in $(2.1)$.

Definition 2.3. Let $\mathbf{o}=\sum_{\ell=0}^{7} o_{\ell} \boldsymbol{e}_{\ell} \in \mathbb{O}$. The column, vectorial or ket representation of $\mathbf{o}$ is $|\mathbf{o}\rangle=\left[\begin{array}{llll}o_{0} & o_{1} & \cdots & o_{7}\end{array}\right]^{T}$.

With this notation, the following theorem enumerates some properties relating these two representations.

Theorem 2.4 (Eganova and Shirokov, [13]; Tian, [31]). Let $\boldsymbol{o}_{\mathbf{1}}, \boldsymbol{o}_{\mathbf{2}}, \boldsymbol{o}_{\mathbf{3}} \in \mathbb{O}$ and $\lambda \in \mathbb{R}$. Then

(i) $\boldsymbol{o}_{\mathbf{1}}=\boldsymbol{o}_{\mathbf{2}}$ if, and only if, $\omega\left(\boldsymbol{o}_{\mathbf{1}}\right)=\omega\left(\boldsymbol{o}_{\mathbf{2}}\right)$,

(ii) $\omega\left(\boldsymbol{o}_{\mathbf{1}}+\boldsymbol{o}_{\mathbf{2}}\right)=\omega\left(\boldsymbol{o}_{\mathbf{1}}\right)+\omega\left(\boldsymbol{o}_{\mathbf{2}}\right)$,

(iii) $\omega\left(\lambda \boldsymbol{o}_{1}\right)=\lambda \omega\left(\boldsymbol{o}_{1}\right)$,

(iv) $\omega(1)=I_{8}$,

(v) $\omega\left(\overline{\boldsymbol{o}_{1}}\right)=\omega\left(\boldsymbol{o}_{\mathbf{1}}\right)^{T}$,

(vi) $\omega\left(\boldsymbol{o}_{1}^{2}\right)=\omega\left(\boldsymbol{o}_{\mathbf{1}}\right)^{2}$,

(vii) $\omega\left(\left(\boldsymbol{o}_{\mathbf{1}} \boldsymbol{o}_{\mathbf{2}}\right) \boldsymbol{o}_{\mathbf{1}}\right)=\omega\left(\boldsymbol{o}_{\mathbf{1}}\right) \omega\left(\boldsymbol{o}_{\mathbf{2}}\right) \omega\left(\boldsymbol{o}_{\mathbf{1}}\right)$,

(viii) $\omega\left(\boldsymbol{o}_{\mathbf{1}} \boldsymbol{o}_{\mathbf{2}}\right)+\omega\left(\boldsymbol{o}_{\mathbf{2}} \boldsymbol{o}_{\mathbf{1}}\right)=\omega\left(\boldsymbol{o}_{\mathbf{1}}\right) \omega\left(\boldsymbol{o}_{\mathbf{2}}\right)+\omega\left(\boldsymbol{o}_{\mathbf{2}}\right) \omega\left(\boldsymbol{o}_{\mathbf{1}}\right)$,

(ix) $\left|\boldsymbol{o}_{\mathbf{1}} \boldsymbol{o}_{\mathbf{2}}\right\rangle=\omega\left(\boldsymbol{o}_{\mathbf{1}}\right)\left|\boldsymbol{o}_{\mathbf{2}}\right\rangle$, 
(x) $\left|\boldsymbol{o}_{1}\left(\boldsymbol{o}_{2} \boldsymbol{o}_{3}\right)\right\rangle=\omega\left(\boldsymbol{o}_{1}\right) \omega\left(\boldsymbol{o}_{2}\right)\left|\boldsymbol{o}_{3}\right\rangle$

An equivalence relation $\sim$ over $\mathbb{O}$ is also defined. For any two octonions $\boldsymbol{O}$ and $\boldsymbol{o}^{\prime}, \boldsymbol{o} \sim \boldsymbol{o}^{\prime}$ if there exists $\sigma \in \mathbb{O}, \sigma \neq 0$, such that $\boldsymbol{o}^{\prime}=\sigma \boldsymbol{o} \sigma^{-1}$. In this case, $\boldsymbol{o}$ and $\boldsymbol{o}^{\prime}$ are said to be similar. The conjugacy class of $\boldsymbol{o}$, denoted by $[\boldsymbol{o}]$, is the set $\{\boldsymbol{x} \in \mathbb{O}: \boldsymbol{x} \sim \boldsymbol{o}\}$.

This equivalence relation is a main concept in the theory of quaternionic and octonionic polynomials. The following result characterizes the conjugacy classes.

Theorem 2.5 (Tian, [31]). Two octonions, $\boldsymbol{o}$ and $\boldsymbol{o}^{\prime}$, are similar if and only if $\operatorname{Re}(\boldsymbol{o})=\operatorname{Re}\left(\boldsymbol{o}^{\prime}\right)$ and $n_{\boldsymbol{o}}=n_{\boldsymbol{o}^{\prime}}$.

Theorem 2.6 (Serôdio, Beites, and Vitória, [29]). Given an octonion $\boldsymbol{o}=$ $\sum_{\ell=0}^{7} o_{\ell} \boldsymbol{e}_{\ell}$, the real matrix $\omega(\boldsymbol{o})$ has two complex eigenvalues, each with multiplicity $4, \lambda=o_{0} \pm i \sqrt{\sum_{\ell=1}^{7} o_{\ell}^{2}}$.

\subsection{Unilateral Octonionic Polynomials}

As the coefficients can be on the left, on the right or on both sides of a variable, there are several ways to define octonionic polynomials. The octonionic polynomials whose coefficients are on the left of the variable are called left unilateral octonionic polynomials. Right unilateral octonionic polynomials are defined in an analogous way. Moreover, all the results for left unilateral polynomials have corresponding results for right unilateral polynomials. For this reason, we restrict our attention to left unilateral octonionic polynomials, by referring to them simply as unilateral octonionic polynomials.

Let $\mathrm{O}[X]$ denote the ring of unilateral polynomials in the variable $x$ over $\mathbb{O}$. Every polynomial $p \in \mathbb{O}[X]$ can be written as $p(x)=\boldsymbol{a}_{m} x^{m}+\boldsymbol{a}_{m-1} x^{m-1}+$ $\cdots+\boldsymbol{a}_{1} x+\boldsymbol{a}_{0}$, for some nonnegative integer $m$, and $\boldsymbol{a}_{r} \in \mathbb{O}, r=0,1, \ldots, m$, where $\boldsymbol{a}_{m} \neq 0$. Under these conditions, $m$ is called the degree of $p$, which will be denoted by $\operatorname{deg}(p)=m$. If $\boldsymbol{a}_{m}=1$ the polynomial is said to be monic; otherwise it is said to be non-monic. For the particular case where the coefficients of the octonionic polynomial are real, the corresponding polynomial ring will be denoted by $\mathbb{R}[X]$.

The equality and the addition of two polynomials are defined in the usual way. Contrarily, the multiplication of two octonionic polynomials is defined in a standard although unexpected way, treating the variable $x$ as real, i.e., commuting with the octonion coefficients. The multiplication of two octonionic polynomials, $p$ and $q$, will be denoted by $p \star q$.

The evaluation of an octonionic polynomial $p$ at an octonion $\boldsymbol{o}$ can only be done after expressing $p$ with all the coefficients at the left of the variable. 
Then, the evaluation of $p$ at $\boldsymbol{o}$ is the result of replacing $x$ by $\boldsymbol{o}$. The evaluation at $\boldsymbol{o}$ is not a ring homomorphism from $\mathbb{O}[X]$ to $\mathbb{O}$.

An octonion $\boldsymbol{o}$ is said to be a zero of $p$ if $p(\boldsymbol{o})=0$. We will denote the set of all the zeros of $p$ by $Z \operatorname{ero}(p)$.

Definition 2.7. Let $p \in \mathbb{O}[X]$. The spectral radius of $p$ is denoted by $\rho(p)$ and is defined by

$$
\rho(p)=\max \left\{\|\boldsymbol{o}\|_{2}: \boldsymbol{o} \in \operatorname{Zero}(p)\right\},
$$

i. e., is the largest of the 2-norm values of the zeros of $p$.

For any $\boldsymbol{o} \in \mathbb{O}$, the real polynomial $\Delta_{\boldsymbol{o}}(x)=(x-\overline{\boldsymbol{o}}) \star(x-\boldsymbol{o})=x^{2}-t_{\boldsymbol{o}} x+n_{\boldsymbol{o}}$ is called the characteristic polynomial of the octonion $\boldsymbol{o}$. Note that $\Delta_{\boldsymbol{o}}$ is an irreducible real quadratic polynomial if $\boldsymbol{o} \in \mathbb{O} \backslash \mathbb{R}$.

Lemma 2.8 (Serôdio, [26]). Let $\boldsymbol{o} \in \mathbb{O}$. Then $\Delta_{\boldsymbol{o}}(\boldsymbol{o})=0$.

From the definitions of conjugacy class and characteristic polynomial of an octonion, it follows that two octonions are similar if and only if they have the same characteristic polynomial.

Given $p(x)=\sum_{r=1}^{m} \boldsymbol{a}_{r} x^{r} \in \mathbb{O}[X]$, we define $\bar{p}(x)=\sum_{r=1}^{m} \overline{\boldsymbol{a}}_{r} x^{r}$ and the normal polynomial of $p$, denoted by $n_{p}$, as

$$
n_{p}(x)=(p \star \bar{p})(x)=(\bar{p} \star p)(x)=\sum_{0 \leq r, s \leq m} \boldsymbol{a}_{r} \overline{\boldsymbol{a}}_{s} x^{r+s} .
$$

Notice that $n_{p} \in \mathbb{R}[X]$ and $\operatorname{deg}\left(n_{p}\right)=2 \operatorname{deg}(p)$.

Remark 2.9. We can write the octonionic polynomial $p$ as

$$
p(x)=\sum_{i=0}^{7} \boldsymbol{e}_{i} p_{i}(x)=p_{0}(x)+\sum_{i=1}^{7} \boldsymbol{e}_{i} p_{i}(x) .
$$

Hence,

$$
\bar{p}(x)=p_{0}(x)-\sum_{i=0}^{7} \boldsymbol{e}_{i} p_{i}(x) .
$$

Multiplying $p$ by $\bar{p}$, it is easy to verify that

$$
n_{p}(x)=(p \star \bar{p})(x)=\sum_{i=0}^{7} p_{i}^{2}(x) .
$$

Two key results on octonionic polynomials are enunciated in what follows. They relate the classes of conjugacy of the zeros of an octonionic polynomial to the zeros of the corresponding normal polynomial. 
Theorem 2.10 (Serôdio, $[26]$ ). Let $p \in \mathbb{O}[X]$ and $\boldsymbol{o} \in \mathbb{O}$. Then $\Delta_{\boldsymbol{o}}$ divides $n_{p}$ if, and only if, there exists at least one $\boldsymbol{o}^{\prime} \sim \boldsymbol{o}$ such that $p\left(\boldsymbol{o}^{\prime}\right)=0$.

Corollary 2.11. The zeros of $p \in \mathbb{O}[X]$ belong to one of the classes $\left[\boldsymbol{o}^{\prime}\right]$, where $\boldsymbol{o}^{\prime}$ is a zero of $n_{p}$.

\subsection{Matricial Norms and Spectral Radius}

Consider the set of nonnegative reals, $\mathbb{R}_{+}$. Let $\mathbb{R}^{m \times m}$ and $\mathbb{R}_{+}^{k \times k}$ denote, respectively, the algebra of real $m \times m$ matrices and the set of all $k \times k$ matrices with entries in $\mathbb{R}_{+}$.

Definition 2.12. The spectral radius of $A \in \mathbb{R}^{m \times m}$ is defined by

$$
\rho(A)=\max _{\lambda \in \sigma(A)}|\lambda|
$$

where $\sigma(A)$ denotes the set of distinct eigenvalues of $A$.

Recall that the set $\mathbb{R}_{+}^{k \times k}$ is partially ordered componentwise, that is, for all $\left[a_{i j}\right],\left[b_{i j}\right] \in \mathbb{R}_{+}^{k \times k},\left[a_{i j}\right] \leq\left[b_{i j}\right]$ if and only if $a_{i j} \leq b_{i j}, i, j \in\{1, \ldots, n\}$.

Definition 2.13. A mapping $\mu: \mathbb{R}^{m \times m} \rightarrow \mathbb{R}_{+}^{k \times k}$ is a matricial norm if, for any $A, B \in \mathbb{R}^{m \times m}$ and $\alpha \in \mathbb{R}$,

(i) $\mu(\alpha A)=|\alpha| \mu(A)$

(ii) $\mu(A+B) \leq \mu(A)+\mu(B)$

(iii) $\mu(A B) \leq \mu(A) \mu(B)$

(iv) $\mu(A) \neq 0$ if $A \neq 0$.

If $k=1$ then $\mu$ is a matrix norm.

Particular classes of matrix norms can be obtained in the following ways:

$$
\|A\|_{1}=\max _{1 \leq j \leq m} \sum_{i=1}^{m}\left|a_{i j}\right|, \quad\|A\|_{\infty}=\max _{1 \leq i \leq m} \sum_{j=1}^{m}\left|a_{i j}\right|, \quad\|A\|_{2}=\sqrt{\rho\left(A^{T} A\right)} .
$$

These matrix norms are vector-induced (or subordinate) and are usually known as 1-norm or column norm, $\infty$-norm or row norm, 2-norm or spectral norm, respectively, [19].

The subsequent result shows how one can generate a matricial norm on $\mathbb{R}^{m \times m}$ starting with a matrix norm on $\mathbb{R}^{m \times m}$. 
Theorem 2.14 (Deutsch, [11]). Let $E_{1}, \ldots, E_{k}$ be the projections associated with a direct-sum decomposition $\mathbb{R}^{n}=X_{1} \oplus \cdots \oplus X_{k}$ of $\mathbb{R}^{n}$, and let $\psi$ be a matrix norm on $\mathbb{R}^{m \times m}$. Then the mapping

$$
\begin{aligned}
& \mu: \mathbb{R}^{m \times m} \rightarrow \mathbb{R}_{+}^{k \times k} \\
& \mu(A)=\left(\psi\left(E_{i} A E_{j}\right)\right)_{i, j=1, \ldots, k}
\end{aligned}
$$

is a matricial norm on $\mathbb{R}^{m \times m}$, called the matricial norm induced by the directsum decomposition $\mathbb{R}^{n}=X_{1} \oplus \cdots \oplus X_{k}$ and the matrix norm $\psi$.

Remark 2.15. In a concrete form, we generate a matricial norm in the following way: given a real $m \times m$ matrix, we partition it into a $k \times k$ block matrix such that the diagonal matrices are square, not necessarily of the same order, and then consider a matrix norm on each block.

We finish this subsection with properties involving matricial norms and spectral radii.

Theorem 2.16 (Deutsch, [11]; Horn and Johnson, [19]). Let $\mu: \mathbb{R}^{m \times m} \rightarrow$ $\mathbb{R}_{+}^{k \times k}$ be a matricial norm and let $\|\cdot\|$ be a matrix norm. Then, for all $A \in \mathbb{R}^{m \times m}$ and for all positive integers $q$,

$$
\rho(A) \leq\left[\rho\left(\mu\left(A^{q}\right)\right)\right]^{1 / q} \text { and } \rho(A) \leq\|A\|_{i}, i=1,2, \infty .
$$

\subsection{Matrix Polynomials}

We end Section 2 focusing on the concept of block companion matrix associated with a matrix polynomial. To this subject has been given considerable attention in the literature and is well-known.

The following definitions and results can be found in [10].

Definition 2.17. Let $A_{0}, A_{1}, \ldots, A_{m} \in \mathbb{R}^{n \times n}$. The matrix function in the variable $X \in \mathbb{R}^{n \times n}$ given by

$$
M(X)=A_{m} X^{m}+A_{m-1} X^{m-1}+\cdots+A_{1} X+A_{0}
$$

is called a matrix polynomial. If $A_{m}=I_{n}$, then $M(X)$ is said to be monic and, for simplicity, it is usual to omit $A_{m}$.

Definition 2.18. Let $M(X)$ be a matrix polynomial. If $X=\lambda I_{n}$, then

$$
M\left(\lambda I_{n}\right)=M(\lambda)=A_{m} \lambda^{m}+A_{m-1} \lambda^{m-1}+\cdots+A_{1} \lambda+A_{0},
$$

is called a lambda-matrix. 
Definition 2.19. Let $M(\lambda)$ be a lambda-matrix. The latent roots of $M(\lambda)$ are the solutions of $\operatorname{det}(M(\lambda))=0$.

Definition 2.20. Let $M(X)=X^{m}+A_{m-1} X^{m-1}+\cdots+A_{1} X+A_{0}$ be a monic matrix polynomial. The matrix

$$
C(M)=\left[\begin{array}{cccc:c}
0_{n} & 0_{n} & \cdots & 0_{n} & -A_{0} \\
\hdashline \overline{I_{n}} & 0_{n} & \cdots & 0_{n} & -A_{1} \\
0_{n} & I_{n} & \cdots & 0_{n} & -A_{2} \\
\vdots & \vdots & \ddots & \vdots & \vdots \\
0_{n} & 0_{n} & \cdots & I_{n} & -A_{m-1}
\end{array}\right]
$$

is called the block companion matrix associated with $M(X)$. For simplicity, we will write $C$ instead of $C(M)$ when there is no doubt which polynomial we are referring to.

In section 3 we will link the next theorem to the octonionic polynomials and take advantage of known results.

Theorem 2.21 (Barnett, [2]; Dennis, Traub and Weber, [10]). Let $C$ be the block companion matrix associated with a monic matrix polynomial $M(X)$. The eigenvalues of $C$ are the latent roots of the associated lambda-matrix $M(\lambda)$.

\section{Main Results on Bounds}

The present section is dedicated to the localization of the zeros of unilateral octonionic polynomials. We will show how this localization problem is related to the localization of latent roots.

\subsection{Octonionic Polynomials and Lambda-Matrices}

We start by associating the octonionic polynomial $p$ with a matrix polynomial $P$ and see how the zeros of $p$ are related to the latent roots of $P$.

Definition 3.1. Let $p \in \mathbb{O}[X]$ be of degree $m$ and given by $p(x)=\boldsymbol{a}_{m} x^{m}+$ $\boldsymbol{a}_{m-1} x^{m-1}+\cdots+\boldsymbol{a}_{0}$. The associated matrix polynomial $P$ is given by

$$
P(X)=A_{m} X^{m}+A_{m-1} X^{m-1}+\cdots+A_{0},
$$

where $A_{i}=\omega\left(\boldsymbol{a}_{i}\right) \in \mathbb{R}^{8 \times 8}, i=0, \ldots, m$, with $\omega$ defined in (2.1).

Lemma 3.2. Let $p \in \mathbb{O}[X]$ and $P$ be its associated matrix polynomial. Then the associated matrix polynomial $\bar{P}$ of $\bar{p} \in \mathbb{O}[X]$ satisfies

$$
\bar{P}(\lambda)=(P(\lambda))^{T} .
$$


Proof. Let $p \in \mathbb{O}[X]$. Writing $p$ as $p(x)=\sum_{i=0}^{7} \boldsymbol{e}_{i} p_{i}(x)$ the result follows directly from property $(\mathrm{v})$ of Theorem 2.4.

Lemma 3.3. Let $p \in \mathbb{O}[X]$ and $P$ be its associated matrix polynomial. If $p(x)=\sum_{i=0}^{7} \boldsymbol{e}_{i} p_{i}(x)$, then

$$
P(\lambda)(P(\lambda))^{T}=\sum_{i=0}^{7} p_{i}^{2}(x) I_{8}
$$

Proof. Straightforward from the multiplication of the matrices $P(\lambda)$ and $\bar{P}(\lambda)$.

Theorem 3.4. Let $p \in \mathbb{O}[X]$, let $P$ be its associated matrix polynomial, and let $\lambda \in \mathbb{C}$. Then there exists an octonion $\boldsymbol{o} \in[\lambda]$ such that $p(\boldsymbol{o})=0$ if and only if $\operatorname{det}(P(\lambda))=0$.

Proof. Let $p \in \mathbb{O}[X]$ be of degree $m$ and the normal polynomial of $p$ be given by

$$
n_{p}(x)=(p \star \bar{p})(x)=b_{2 m} x^{2 m}+b_{2 m-1} x^{2 m-1}+\cdots+b_{1} x+b_{0},
$$

where $b_{i} \in \mathbb{R}, i=0, \ldots, 2 m$.

Thus $N_{p}$, the associated matrix polynomial of $n_{p}$,

$$
N_{p}(X)=B_{2 m} X^{2 m}+B_{2 m-1} X^{2 m-1}+\cdots+B_{1} X+B_{0},
$$

is a polynomial whose coefficients are scalar matrices. Hence the lambdamatrix $N_{p}(\lambda)$, corresponding to $N_{p}(X)$, is

$$
\begin{aligned}
N_{p}(\lambda) & =B_{2 m} \lambda^{2 m}+B_{2 m-1} \lambda^{2 m-1}+\cdots+B_{0} \\
& =b_{2 m} \lambda^{2 m} I_{8}+b_{2 m-1} \lambda^{2 m-1} I_{8}+\cdots+b_{0} I_{8} \\
& =n_{p}(\lambda) I_{8} .
\end{aligned}
$$

On the other hand, by Lemmas 3.2 and 3.3 and Remark 2.9, we obtain

$$
N_{p}(\lambda)=P(\lambda) \bar{P}(\lambda)
$$

We have successively,

$$
\begin{aligned}
\operatorname{det}\left(N_{p}(\lambda)\right) & =\operatorname{det}(P(\lambda) \bar{P}(\lambda)) \\
\operatorname{det}\left(n_{p}(\lambda) I\right) & =\operatorname{det}\left(P(\lambda) P(\lambda)^{T}\right) \\
\left(n_{p}(\lambda)\right)^{8} & =\operatorname{det}(P(\lambda))^{2} .
\end{aligned}
$$


Hence,

$$
\operatorname{det}(P(\lambda))= \pm\left(n_{p}(\lambda)\right)^{4}
$$

Since the leading coefficients of $\operatorname{det}(P(\lambda))$ and $n_{p}(\lambda)$ are positive, we conclude that $\operatorname{det}(P(\lambda))=\left(n_{p}(\lambda)\right)^{4}$, from what follows the result.

Corollary 3.5. Let $p \in \mathbb{O}[X], P$ its associated matrix polynomial and $\boldsymbol{o} \in \mathbb{O}$ such that $\boldsymbol{o} \in \operatorname{Zero}(p)$. The following statements are equivalent:

1. $\boldsymbol{o} \in[\lambda]$

2. $\operatorname{det}(P(\lambda))=0$;

3. $\operatorname{det}(\lambda I-C(\widetilde{P}))=0$, where $\widetilde{P}$ is the corresponding monic matrix polynomial of $P$.

Proof. A direct consequence of Theorem 2.21 and Theorem 3.4.

Corollary 3.6. Let $p \in \mathbb{O}[X]$ and $C$ be the companion matrix of $\widetilde{P}=$ $A_{m}^{-1} P(X)$, where $P$ is the associated matrix polynomial of $p$. Then, $\rho(p)=$ $\rho(C)$.

Proof. A direct consequence of the previous Corollary.

\subsection{Localization and Upper Bounds for the Zeros of Unilateral Octonionic Polynomials}

Taking into account Section 3.1, we can claim that locating the zeros of an octonionic polynomial $p$ is equivalent to locating the latent roots of its associated lambda-matrix $P(\lambda)$. This implies that all research developed for the localization of latent roots of lambda-matrices can be applied to the localization of the zeros of octonionic polynomials without restriction. Even more, this problem is less restrictive since the leading matrix coefficient is always invertible. It is worth mentioning that this is also valid for quaternionic polynomials.

Bounds for the latent roots of lambda-matrices have been widely studied and many localization theorems have been published [18, 23, 32, 33]. Some results for locating the latent roots are extended from well known results for complex polynomials $[5,24]$. Nowadays some results for complex polynomials are still being obtained $[7,9,12,22,30,34]$.

In the following subsections we present some results for octonionic polynomials extended from matrix polynomials, which in turn have been extended from complex polynomials. For this reason, and taking into account previous sections, the proofs of the results undermentioned follow the proofs for 
the matricial case. Therefore, almost all theorems will be presented without proof.

The choice of these theorems is only illustrative of their applicability, and they were chosen in order to compare the bounds.

\subsubsection{Cauchy-like and Pellet-like results}

The well known Cauchy's Theorem in [5] can now be extended to octonionic polynomials. We present a proof just to illustrate the above idea.

Theorem 3.7. Let $p \in \mathbb{O}[X]$, of degree $m$, be given by $p(x)=\boldsymbol{a}_{m} x^{m}+$ $\boldsymbol{a}_{m-1} x^{m-1}+\cdots+\boldsymbol{a}_{1} x+\boldsymbol{a}_{0}$. Then $Z \operatorname{ero}(p) \subset\left\{\boldsymbol{o} \in \mathbb{O}:\|\boldsymbol{o}\|_{2} \leq R_{i}\right\}$, where $R_{i}$ is the unique positive solution of the real equation

$$
x^{m}-\frac{\left\|\boldsymbol{a}_{m-1}\right\|_{i}}{\left\|\boldsymbol{a}_{m}\right\|_{i}} x^{m-1}-\cdots-\frac{\left\|\boldsymbol{a}_{1}\right\|_{i}}{\left\|\boldsymbol{a}_{m}\right\|_{i}} x-\frac{\left\|\boldsymbol{a}_{0}\right\|_{i}}{\left\|\boldsymbol{a}_{m}\right\|_{i}}=0, \quad i=1,2 .
$$

Proof. Given $p(x)=\boldsymbol{a}_{m} x^{m}+\boldsymbol{a}_{m-1} x^{m-1}+\cdots+\boldsymbol{a}_{1} x+\boldsymbol{a}_{0} \in \mathbb{O}[X]$, we construct the respective monic associated matrix polynomial

$$
\widetilde{P}(X)=X^{m}+\widetilde{A}_{m-1} X^{m-1}+\cdots+\widetilde{A}_{0}
$$

By Corollary 3.6, the spectral radius of $p$ is equal to the spectral radius of $C$, where $C$ is the companion matrix of $\widetilde{P}$, i.e, $\rho(p)=\rho(C)$.

In addition, by Theorem 2.16, $\rho(C) \leq \rho(\mu(C))$, where $\mu(\cdot)$ is a matricial norm. Considering the matricial norm that applies the matrix norm $\|\cdot\|_{i}$, for $i=1,2$, to each $8 \times 8$ block, and taking into consideration that $\left\|A_{m}^{-1} A_{\ell}\right\|_{i}=$ $\frac{\left\|A_{\ell}\right\|_{i}}{\left\|A_{m}\right\|_{i}}=\frac{\left\|a_{\ell}\right\|_{i}}{\left\|a_{m}\right\|_{i}}$, for $\ell=0, \ldots, m-1$, then

$$
\mu(C)=\left[\begin{array}{cccc}
0 & \cdots & 0 & \left\|a_{0}\right\|_{i} /\left\|a_{m}\right\|_{i} \\
1 & \cdots & 0 & \left\|a_{1}\right\|_{i} /\left\|a_{m}\right\|_{i} \\
\vdots & \ddots & \vdots & \vdots \\
0 & \cdots & 1 & \left\|a_{m-1}\right\|_{i} /\left\|a_{m}\right\|_{i}
\end{array}\right] .
$$

This matrix is the companion matrix of the real polynomial in the first member of (3.1) which has a unique positive zero $R_{i}$. Hence, $\rho(p)=\rho(C) \leq$ $\rho(\mu(C))=R_{i}$.

The interesting Pellet's theorem in [23], which determines regions of exclusion, can also be extended to octonionic polynomials. Unlike Cauchy's theorem, Pellet's theorem does not always bear fruits. 
Theorem 3.8. Let $p \in \mathbb{O}[X]$ be given by

$$
p(x)=\boldsymbol{a}_{m} x^{m}+\boldsymbol{a}_{m-1} x^{m-1}+\cdots+\boldsymbol{a}_{1} x+\boldsymbol{a}_{0},
$$

with $\boldsymbol{a}_{m} \boldsymbol{a}_{0} \boldsymbol{a}_{k} \neq 0$ and $m \geq 3$. Let $1 \leq k \leq m-1$, and let $p_{k} \in \mathbb{R}[X]$ be given by

$$
\begin{aligned}
p_{k}(x)= & \left\|\boldsymbol{a}_{m}\right\|_{2} x^{m}+\left\|\boldsymbol{a}_{m-1}\right\|_{2} x^{m-1}+\cdots+\left\|\boldsymbol{a}_{k+1}\right\|_{2} x^{k+1}- \\
& -\left\|\boldsymbol{a}_{k}\right\|_{2} x^{k}+\left\|\boldsymbol{a}_{k-1}\right\|_{2} x^{k-1}+\cdots+\left\|\boldsymbol{a}_{0}\right\|_{2}
\end{aligned}
$$

If $p_{k}$ has two distinct positive zeros $x_{1}$ and $x_{2}$ with $x_{1}<x_{2}$, then $p$ has exactly $k$ zeros in or on the 7-sphere $|x|=x_{1}$ and no zeros in the 7-spherical shell $x_{1}<|x|<x_{2}$.

The next theorem is an extension to octonionic polynomials of a theorem that can be found in Marden [22].

Theorem 3.9. Let $p \in \mathbb{O}[X]$ be of degree $m$ and given by $p(x)=\boldsymbol{a}_{m} x^{m}+$ $\boldsymbol{a}_{m-1} x^{m-1}+\cdots+\boldsymbol{a}_{1} x+\boldsymbol{a}_{0}$. If $\left\|\boldsymbol{a}_{k}\right\|_{2}<\left\|\boldsymbol{a}_{m}\right\|_{2}, k=0,1, \ldots, m-1$, then $Z \operatorname{ero}(p) \subset\left\{\boldsymbol{o} \in \mathbb{O}:\|\boldsymbol{o}\|_{2} \leq 2\right\}$.

\subsubsection{Other Bounds}

In this subsection, generalizations to octonionic polynomials of complex and matrix polynomials results are presented. The results for complex and matrix polynomials can be found in the work of Deutsch on applications of matricial norms to complex polynomials and in the work of Vitória on applications of matricial norms to lambda matrices, respectively. When not mentioned, the octonion norm can be the norm 1 or the norm 2 .

These first two theorems are extensions to octonionic polynomials of results that can be found in Deutsch [12].

Theorem 3.10. Let $p \in \mathbb{O}[X]$ be of degree $m$ and given by $p(x)=\boldsymbol{a}_{m} x^{m}+$ $\boldsymbol{a}_{m-1} x^{m-1}+\cdots+\boldsymbol{a}_{1} x+\boldsymbol{a}_{0}$. If $k_{0}, k_{1}, \ldots, k_{m-2}$ are arbitrary positive numbers, then

$$
\begin{aligned}
& \rho(p) \leq \frac{1}{2}\left[\beta+\frac{\left\|\boldsymbol{a}_{m-1}\right\|_{i}}{\left\|\boldsymbol{a}_{m}\right\|_{i}}+\sqrt{\left(\frac{\left\|\boldsymbol{a}_{m-1}\right\|_{i}}{\left\|\boldsymbol{a}_{m}\right\|_{i}}-\beta\right)^{2}+\frac{4 \gamma k_{m-2}}{\left\|\boldsymbol{a}_{m}\right\|_{i}}}\right] \\
& \rho(p) \leq \max \left\{\beta+k_{m-2}, \frac{\gamma+\left\|\boldsymbol{a}_{m-1}\right\|_{i}}{\left\|\boldsymbol{a}_{m}\right\|_{i}}\right\} \\
& \rho(p) \leq \sqrt{\beta^{2}+k_{m-2}^{2}+\frac{\gamma^{2}+\left\|\boldsymbol{a}_{m-1}\right\|_{i}^{2}}{\left\|\boldsymbol{a}_{m}\right\|_{i}^{2}}}
\end{aligned}
$$


where $\beta=\max \left\{\frac{k_{0}}{k_{1}}, \frac{k_{1}}{k_{2}}, \ldots, \frac{k_{m-3}}{k_{m-2}}\right\}, \gamma=\max \left\{\frac{\left\|\boldsymbol{a}_{0}\right\|_{i}}{k_{0}}, \frac{\left\|\boldsymbol{a}_{1}\right\|_{i}}{k_{1}}, \ldots, \frac{\left\|\boldsymbol{a}_{m-2}\right\|_{i}}{k_{m-2}}\right\}$, and $i \in\{1,2\}$.

If we take $k_{0}=\cdots=k_{m-2}=1$ in Theorem 3.10 we obtain the following.

Corollary 3.11. Let $p \in \mathbb{O}[X]$ be of degree $m$ and given by $p(x)=\boldsymbol{a}_{m} x^{m}+$ $\boldsymbol{a}_{m-1} x^{m-1}+\cdots+\boldsymbol{a}_{1} x+\boldsymbol{a}_{0}$. Then

$$
\begin{aligned}
\rho(p) & \leq \frac{1}{2}\left[1+\frac{\left\|\boldsymbol{a}_{m-1}\right\|_{i}}{\left\|\boldsymbol{a}_{m}\right\|_{i}}+\sqrt{\left(\frac{\left\|\boldsymbol{a}_{m-1}\right\|_{i}}{\left\|\boldsymbol{a}_{m}\right\|_{i}}-1\right)^{2}+\frac{4 M}{\left\|\boldsymbol{a}_{m}\right\|_{i}}}\right] \\
\rho(p) & \leq \max \left\{2, \frac{\left\|\boldsymbol{a}_{0}\right\|_{i}+\left\|\boldsymbol{a}_{m-1}\right\|_{i}}{\left\|\boldsymbol{a}_{m}\right\|_{i}}, \ldots, \frac{\left\|\boldsymbol{a}_{m-2}\right\|_{i}+\left\|\boldsymbol{a}_{m-1}\right\|_{i}}{\left\|\boldsymbol{a}_{m}\right\|_{i}}\right\} \\
\rho(p) & \leq \sqrt{2+\frac{M^{2}+\left\|\boldsymbol{a}_{m-1}\right\|_{i}^{2}}{\left\|\boldsymbol{a}_{m}\right\|_{i}^{2}}}
\end{aligned}
$$

where $M=\max \left\{\left\|\boldsymbol{a}_{0}\right\|_{i},\left\|\boldsymbol{a}_{1}\right\|_{i}, \ldots,\left\|\boldsymbol{a}_{m-2}\right\|_{i}\right\}$ and $i \in\{1,2\}$.

If we take $k_{\ell}=\frac{\left\|\boldsymbol{a}_{\ell+1}\right\|_{i}}{\left\|\boldsymbol{a}_{m}\right\|_{i}}$, for $\ell=0, \ldots, m-3$ in Theorem 3.10 we obtain the following.

Corollary 3.12. Let $p \in \mathbb{O}[X]$ be of degree $m$ and given by $p(x)=\boldsymbol{a}_{m} x^{m}+$ $\boldsymbol{a}_{m-1} x^{m-1}+\cdots+\boldsymbol{a}_{1} x+\boldsymbol{a}_{0}$. If $\boldsymbol{a}_{j} \neq 0, j=0,1, \ldots, m-1$, then

$$
\begin{aligned}
\rho(p) & \leq \frac{1}{2}\left[\beta^{\prime}+\frac{\left\|\boldsymbol{a}_{m-1}\right\|_{i}}{\left\|\boldsymbol{a}_{m}\right\|_{i}}+\sqrt{\left(\frac{\left\|\boldsymbol{a}_{m-1}\right\|_{i}}{\left\|\boldsymbol{a}_{m}\right\|_{i}}-\beta^{\prime}\right)^{2}+4 \gamma^{\prime}\left\|\boldsymbol{a}_{m-1}\right\|_{i}}\right] \\
\rho(p) & \leq \frac{\left\|\boldsymbol{a}_{m-1}\right\|_{i}}{\left\|\boldsymbol{a}_{m}\right\|_{i}}+\gamma^{\prime} \\
\rho(p) & \leq \sqrt{2 \frac{\left\|\boldsymbol{a}_{m-1}\right\|_{i}^{2}}{\left\|\boldsymbol{a}_{m}\right\|_{i}^{2}}+\beta^{\prime 2}+\gamma^{\prime 2}}
\end{aligned}
$$

where

$$
\begin{aligned}
& \beta^{\prime}=\max \left\{\frac{\left\|\boldsymbol{a}_{1}\right\|_{i}}{\left\|\boldsymbol{a}_{2}\right\|_{i}}, \frac{\left\|\boldsymbol{a}_{2}\right\|_{i}}{\left\|\boldsymbol{a}_{3}\right\|_{i}}, \ldots, \frac{\left\|\boldsymbol{a}_{m-2}\right\|_{i}}{\left\|\boldsymbol{a}_{m-1}\right\|_{i}}\right\}, \\
& \gamma^{\prime}=\max \left\{\frac{\left\|\boldsymbol{a}_{0}\right\|_{i}}{\left\|\boldsymbol{a}_{1}\right\|_{i}}, \frac{\left\|\boldsymbol{a}_{1}\right\|_{i}}{\left\|\boldsymbol{a}_{2}\right\|_{i}}, \ldots, \frac{\left\|\boldsymbol{a}_{m-2}\right\|_{i}}{\left\|\boldsymbol{a}_{m-1}\right\|_{i}}\right\},
\end{aligned}
$$

and $i \in\{1,2\}$. 
The following result uses only one parameter $t$.

Theorem 3.13. Let $p \in \mathbb{O}[X]$ be of degree $m$ and given by $p(x)=\boldsymbol{a}_{m} x^{m}+$ $\boldsymbol{a}_{m-1} x^{m-1}+\cdots+\boldsymbol{a}_{1} x+\boldsymbol{a}_{0}$. If $t$ is an arbitrary positive number, then

$$
\begin{aligned}
\rho(p) & \leq \frac{1}{2}\left[t+\frac{\left\|\boldsymbol{a}_{m-1}\right\|_{i}}{\left\|\boldsymbol{a}_{m}\right\|_{i}}+\sqrt{\left(\frac{\left\|\boldsymbol{a}_{m-1}\right\|_{i}}{\left\|\boldsymbol{a}_{m}\right\|_{i}}-t\right)^{2}+\frac{4 \delta t}{\left\|\boldsymbol{a}_{m}\right\|_{i}}}\right] \\
\rho(p) & \leq \max \left\{2 t, \max \left\{\frac{\delta+\left\|\boldsymbol{a}_{j}\right\|_{i}}{\left\|\boldsymbol{a}_{m}\right\|_{i}}: j=0, \ldots, m-1\right\}\right\}, \\
\rho(p) & \leq \sqrt{2 t^{2}+\frac{\delta^{2}+\left\|\boldsymbol{a}_{m-1}\right\|_{i}^{2}}{\left\|\boldsymbol{a}_{m}\right\|_{i}^{2}}}
\end{aligned}
$$

where $\delta=\max \left\{\frac{\left\|\boldsymbol{a}_{0}\right\|_{i}}{t^{m-1}}, \frac{\left\|\boldsymbol{a}_{1}\right\|_{i}}{t^{m-2}}, \ldots, \frac{\left\|\boldsymbol{a}_{m-2}\right\|_{i}}{t}\right\}$ and $i \in\{1,2\}$.

If we take $t=\frac{\left\|\boldsymbol{a}_{m-1}\right\|_{i}}{\left\|\boldsymbol{a}_{m}\right\|_{i}}$ in Theorem 3.13 we obtain the following.

Corollary 3.14. Let $p \in \mathbb{O}[X]$ be of degree $m$ and given by $p(x)=\boldsymbol{a}_{m} x^{m}+$ $\boldsymbol{a}_{m-1} x^{m-1}+\cdots+\boldsymbol{a}_{1} x+\boldsymbol{a}_{0}$. If $\boldsymbol{a}_{m-1} \neq 0$, then

$$
\begin{aligned}
\rho(p) & \leq \frac{\left\|\boldsymbol{a}_{m-1}\right\|_{i}}{\left\|\boldsymbol{a}_{m}\right\|_{i}}+\sqrt{\delta^{\prime}} \\
\rho(p) & \leq \frac{\left\|\boldsymbol{a}_{m-1}\right\|_{i}}{\left\|\boldsymbol{a}_{m}\right\|_{i}}+\max \left\{\delta^{\prime} \frac{\left\|\boldsymbol{a}_{m}\right\|_{i}}{\left\|\boldsymbol{a}_{m-1}\right\|_{i}}, \frac{\left\|\boldsymbol{a}_{m-1}\right\|_{i}}{\left\|\boldsymbol{a}_{m}\right\|_{i}}\right\} \\
\rho(p) & \leq \sqrt{3\left(\frac{\left\|\boldsymbol{a}_{m-1}\right\|_{i}}{\left\|\boldsymbol{a}_{m}\right\|_{i}}\right)^{2}+\left(\frac{\delta^{\prime}\left\|\boldsymbol{a}_{m}\right\|_{i}}{\left\|\boldsymbol{a}_{m-1}\right\|_{i}}\right)^{2}},
\end{aligned}
$$

where $\delta^{\prime}=\max \left\{\frac{\left\|\boldsymbol{a}_{m}\right\|_{i}^{m-3}\left\|\boldsymbol{a}_{0}\right\|_{i}}{\left\|\boldsymbol{a}_{m-1}\right\|_{i}^{m-2}}, \frac{\left\|\boldsymbol{a}_{m}\right\|_{i}^{m-4}\left\|\boldsymbol{a}_{1}\right\|_{i}}{\left\|\boldsymbol{a}_{m-1}\right\|_{i}^{m-3}}, \ldots, \frac{\left\|\boldsymbol{a}_{m-2}\right\|_{i}}{\left\|\boldsymbol{a}_{m}\right\|_{i}}\right\}$ and $i \in\{1,2\}$.

If we take $t=N$ in Theorem 3.13, where $N$ is defined by

$$
N=\max \left\{\left(\frac{\left\|\boldsymbol{a}_{j}\right\|_{i}}{\left\|\boldsymbol{a}_{m}\right\|_{i}}\right)^{\frac{1}{m-j}}: j=0, \ldots, m-2\right\}
$$

we obtain the following.

Corollary 3.15. Let $p \in \mathbb{O}[X]$ be of degree $m$ and given by $p(x)=\boldsymbol{a}_{m} x^{m}+$ 
$\boldsymbol{a}_{m-1} x^{m-1}+\cdots+\boldsymbol{a}_{1} x+\boldsymbol{a}_{0}$. Then

$$
\begin{aligned}
& \rho(p) \leq \frac{1}{2}\left[N+\frac{\left\|\boldsymbol{a}_{m-1}\right\|_{i}}{\left\|\boldsymbol{a}_{m}\right\|_{i}}+\sqrt{\left(\frac{\left\|\boldsymbol{a}_{m-1}\right\|_{i}}{\left\|\boldsymbol{a}_{m}\right\|_{i}}-N\right)^{2}+4 N^{2}}\right] \\
& \rho(p) \leq N+\max \left\{N, \frac{\left\|\boldsymbol{a}_{m-1}\right\|_{i}}{\left\|\boldsymbol{a}_{m}\right\|_{i}}\right\} \\
& \rho(p) \leq \sqrt{3 N^{2}+\left(\frac{\left\|\boldsymbol{a}_{m-1}\right\|_{i}}{\left\|\boldsymbol{a}_{m}\right\|_{i}}\right)^{2}}
\end{aligned}
$$

where $N$ is given by (3.17) and $i \in\{1,2\}$.

The next theorem is an extension to octonionic polynomials of a generalization of a result that can be found in Vitória [33].

Theorem 3.16. Let $p \in \mathbb{O}[X]$ be of degree $m$ and given by $p(x)=\boldsymbol{a}_{m} x^{m}+$ $\boldsymbol{a}_{m-1} x^{m-1}+\cdots+\boldsymbol{a}_{1} x+\boldsymbol{a}_{0}$, with $\boldsymbol{a}_{0} \neq 0$ and $m \geq 3$. Let $C$ be the companion matrix of $\widetilde{P}=A_{m}^{-1} P(X)$, where $P$ is the associated matrix polynomial of $p$. Then

$$
\rho(p) \leq \sqrt{\frac{\delta_{\kappa, i}+\beta_{\kappa, i}+\sqrt{\left(\delta_{\kappa, i}-\beta_{\kappa, i}\right)^{2}+4 \alpha_{\kappa, i} \gamma_{\kappa, i}}}{2}}
$$

where

$$
C^{2}=\left[\begin{array}{ll}
\delta_{\kappa, i} & \alpha_{\kappa, i} \\
\gamma_{\kappa, i} & \beta_{\kappa, i}
\end{array}\right]
$$

is partitioned into square diagonal blocks through column $\kappa \in\{8,16, \ldots, 8(m-$ $2)\}$ and taking the norm $\|\cdot\|_{i}, i \in\{1,2, \infty\}$, of these blocks.

Proof. Let $\widetilde{P}=A_{m}^{-1} P(X)$, where $P$ is the associated matrix polynomial of $p$. By Corollary 3.6, we know that $\rho(p)=\rho(C)$. Using Theorem 2.16 with $q=2$, we obtain

$$
\rho(p) \leq \sqrt{\rho\left(\mu\left(C^{2}\right)\right)},
$$

where $\mu$ is a matricial norm. Taking $\mu$ in such a way that it partitions the matrix $C^{2}$ into a $2 \times 2$ matrix preserving the octonion blocks, i.e., taking $\kappa \in\{8,16, \ldots, 8(m-2)\}$, we obtain the partitioned matrix

$$
C^{2}=\left[\begin{array}{ll}
\delta_{\kappa, i} & \alpha_{\kappa, i} \\
\gamma_{\kappa, i} & \beta_{\kappa, i}
\end{array}\right]
$$

whose greatest eigenvalue is $\frac{\delta_{\kappa, i}+\beta_{\kappa, i}+\sqrt{\left(\delta_{\kappa, i}-\beta_{\kappa, i}\right)^{2}+4 \alpha_{\kappa, i} \gamma_{\kappa, i}}}{2}$. From here we obtain (3.21). 
Corollary 3.17. Let $p \in \mathbb{O}[X]$ be of degree $m$ and given by $p(x)=\boldsymbol{a}_{m} x^{m}+$ $\boldsymbol{a}_{m-1} x^{m-1}+\cdots+\boldsymbol{a}_{1} x+\boldsymbol{a}_{0}$, with $m \geq 3$, and $P(X)=A_{m} X^{m}+\cdots+A_{0}$ is the associated matrix polynomial of $p$. Then

$$
\rho(p) \leq\left(\frac{\beta_{i}+\sqrt{\beta_{i}^{2}+4 \alpha_{i}}}{2}\right)^{1 / 2} \quad(i=1,2, \infty),
$$

where

$$
\alpha_{i}=\left\|-\widetilde{A}_{0} \quad \widetilde{A}_{0} \widetilde{A}_{2}\right\|_{i}, \beta_{i}=\left\|\begin{array}{cc}
-\widetilde{A}_{1} & -\widetilde{A}_{0}+\widetilde{A}_{1} \widetilde{A}_{2} \\
-\widetilde{A}_{2} & -\widetilde{A}_{1}+\widetilde{A}_{2}^{2}
\end{array}\right\|_{i},
$$

if $m=3$, and

$$
\begin{aligned}
& \alpha_{i}=\left\|\begin{array}{c:cc}
0_{2, m-4} & -\widetilde{A}_{0} & \widetilde{A}_{0} \widetilde{A}_{m-1} \\
& -\widetilde{A}_{1} & -\widetilde{A}_{0}+\widetilde{A}_{1} \widetilde{A}_{m-1}
\end{array}\right\|_{i}, \\
& \beta_{i}=\left\|\begin{array}{|c:cc}
0_{2, m-4} & -\widetilde{A}_{2} & -\widetilde{A}_{1}+\widetilde{A}_{2} \widetilde{A}_{m-1} \\
& -\widetilde{A}_{3} & -\widetilde{A}_{2}+\widetilde{A}_{3} \widetilde{A}_{m-1} \\
I_{m-4} & -\widetilde{A}_{m-1} & -\widetilde{A}_{m-2}+\widetilde{A}_{m-1} \widetilde{A}_{m-1}
\end{array}\right\|_{i}
\end{aligned}
$$

if $m>3$, with $\widetilde{A}_{\ell}=A_{m}^{-1} A_{\ell}, \ell \in\{0, \ldots, m-1\}$.

Proof. Take $\kappa=8$ and $\kappa=16$ in Theorem 3.16 for $m=3$ and $m>3$, respectively, where $\delta_{\kappa, i}=0$ and $\gamma_{\kappa, i}=1$ with $i \in\{1,2, \infty\}$.

\subsection{Lower Bounds for the Zeros of Unilateral Octonionic Polyno- mials}

As a consequence of the results presented in subsection 3.2, lower bounds and exclusion regions for the zeros of unilateral octonionic polynomials are obtained in this subsection. This is accomplished through a connection of the zeros of a reciprocal polynomial with the zeros of its original polynomial.

Let $p \in \mathbb{O}[X]$, of degree $m$, be given by

$$
p(x)=\boldsymbol{a}_{m} x^{m}+\boldsymbol{a}_{m-1} x^{m-1}+\cdots+\boldsymbol{a}_{1} x+\boldsymbol{a}_{0}, \text { where } \boldsymbol{a}_{0} \neq 0 .
$$

Consider the reciprocal polynomial of $p, p_{r} \in \mathbb{O}[X]$ defined by $x^{m} \star p\left(x^{-1}\right)$, that is,

$$
p_{r}(x)=\boldsymbol{a}_{0} x^{m}+\boldsymbol{a}_{1} x^{m-1}+\cdots+\boldsymbol{a}_{m-1} x+\boldsymbol{a}_{m} .
$$

The zeros of $p_{r}$ are the reciprocals of the zeros of $p$. To obtain a lower bound for the zeros of $p$ is equivalent to obtaining a upper bound for the zeros of $p_{r}$. 


\subsection{Numerical Experiments}

We conclude with some illustrative examples of the regions (inclusion and exclusion) and the bounds (lower and upper) presented in Subsections 3.2 and 3.3. We will consider three examples. Almost all formulas are comparable, as shown in Example 3.1. Indeed, the only one that is not comparable is the Pellet-like result (Example 3.2). In Example 3.3, we simulate over a set of polynomials applying Vitória-like results with several partitions.

Example 3.1. For this example we could have constructed an octonionic polynomial with a prescribed set of zeros (see [27] for how to construct an octonionic polynomial with prescribed conditions), but this would probably imply a messy set of coefficients. Since we are not interested in the zeros but in a bound for them, we prevailed the coefficients choosing them with integer imaginary parts.

The octonionic polynomial $p_{1}(x)=\boldsymbol{a}_{3} x^{3}+\boldsymbol{a}_{2} x^{2}+\boldsymbol{a}_{1} x+\boldsymbol{a}_{0}$, with coefficients

$$
\begin{aligned}
& \boldsymbol{a}_{3}=1+2 \boldsymbol{j}-5 \boldsymbol{i} \boldsymbol{k}+\boldsymbol{j} \boldsymbol{k}-4 \boldsymbol{i} \boldsymbol{j} \boldsymbol{k} \\
& \boldsymbol{a}_{2}=-\boldsymbol{i}+\boldsymbol{j}+\boldsymbol{k}-\boldsymbol{i} \boldsymbol{k}-\boldsymbol{j} \boldsymbol{k}-\boldsymbol{i} \boldsymbol{j} \boldsymbol{k} \\
& \boldsymbol{a}_{1}=6+3 \boldsymbol{k} \\
& \boldsymbol{a}_{0}=5 \boldsymbol{i}-4 \boldsymbol{k}+2 \boldsymbol{i} \boldsymbol{j} \boldsymbol{k},
\end{aligned}
$$

has exactly three zeros. They belong to the following approximate conjugacy classes

$$
\begin{gathered}
{[0.723477+0.480511 i]} \\
{[-0.0121304+0.965987 i]} \\
{[-0.924139+0.711349 i] .}
\end{gathered}
$$

This means that the greatest 2-norm value equals approximately 1.166212 and the smallest approximately 0.868510 . We choose the coefficients so that Theorem 3.9 could be applied, and indeed the spectral radius is approximately 1.166212 , which is less than 2 , as expected.

The upper bounds given by (3.2) - (3.22) and the corresponding lower bounds are presented in Table 2, where the best bounds are highlighted for each of the two norms used for the octonions. The values of $k_{0}$ and $k_{1}$ for (3.2), (3.3), and (3.4) and those of $t$ for (3.11), (3.12) and (3.13) were found using a computer. We chose an initial value for $t$ and an initial step and went in the direction which decreased the bound value. When the neighbour values were both greater, the step was reduced. This process was maintained until we obtained a reasonable value for $t$. The same was done for the $k \mathrm{~s}$, but at this time the search was performed in two dimensions. These values are presented in Table 1, although they may not correspond to the best values. 
BOUNDS FOR THE ZEROS OF UNILATERAL OCTONIONIC

\begin{tabular}{|c|c|c|c|c|c|c|}
\hline & & & & $\mathrm{rm}$ & & $\mathrm{rm}$ \\
\hline Result & Ref. & Bound & $k_{0}$ & $k_{1}$ & $k_{0}$ & $k_{1}$ \\
\hline \multirow{6}{*}{ Theorem 3.10} & \multirow{2}{*}{$(3.2)$} & Lower & 10.000000 & 10.000000 & 10.091000 & 10.036100 \\
\hline & & Upper & 9.197100 & 11.001000 & 9.290100 & 10.901000 \\
\hline & \multirow{2}{*}{$(3.3)$} & Lower & 1.000000 & 1.000000 & 1.000000 & 0.900000 \\
\hline & & Upper & 0.698977 & 0.836049 & 0.700000 & 0.800000 \\
\hline & \multirow{2}{*}{$(3.4)$} & Lower & 1.100000 & 1.000000 & 1.014600 & 1.007290 \\
\hline & & Upper & 0.900000 & 1.000000 & 0.985600 & 0.992790 \\
\hline & & & \multicolumn{2}{|c|}{$t$} & \multicolumn{2}{|c|}{$t$} \\
\hline \multirow{6}{*}{ Theorem 3.13} & \multirow{2}{*}{$(3.11)$} & Lower & \multirow{2}{*}{\multicolumn{2}{|c|}{$\begin{array}{l}1.000000 \\
0.836044\end{array}$}} & \multirow{2}{*}{\multicolumn{2}{|c|}{$\begin{array}{l}1.005432 \\
0.852234\end{array}$}} \\
\hline & & Upper & & & & \\
\hline & \multirow{2}{*}{$(3.12)$} & Lower & \multirow{2}{*}{\multicolumn{2}{|c|}{$\begin{array}{l}1.000000 \\
0.836049\end{array}$}} & \multicolumn{2}{|c|}{1.005458} \\
\hline & & Upper & & & \multicolumn{2}{|c|}{0.852244} \\
\hline & \multirow{2}{*}{ (3.13) } & Lower & & \multirow{2}{*}{\multicolumn{2}{|c|}{$\begin{array}{l}1.007278 \\
0.992767\end{array}$}} \\
\hline & & Upper & \multicolumn{2}{|c|}{0.945831} & & \\
\hline
\end{tabular}

Table 1: Values of $k_{0}, k_{1}$, and $t$ for formulas (3.2)-(3.4) and (3.11)-(3.13).

\begin{tabular}{|c|c|c|c|c|c|}
\hline \multirow{2}{*}{ Result } & \multirow{2}{*}{ Ref. } & \multicolumn{2}{|c|}{ Lower bound } & \multicolumn{2}{c|}{ Upper bound } \\
\cline { 3 - 6 } & & 1-norm & 2 -norm & 1-norm & 2 -norm \\
\hline Theorem 3.7 & $(3.1)$ & $\mathbf{0 . 6 2 0 4 8 8}$ & 0.618505 & $\mathbf{1 . 3 9 3 8 0 2}$ & $\mathbf{1 . 4 7 2 7 8 4}$ \\
\hline & $(3.2)$ & 0.500000 & 0.497286 & 1.672098 & 1.704487 \\
Theorem 3.10 & $(3.3)$ & 0.500000 & 0.494565 & 1.672098 & 1.755141 \\
& $(3.4)$ & 0.497906 & 0.497285 & 1.704975 & 1.756271 \\
\hline & $(3.5)$ & 0.500000 & 0.497282 & 1.689226 & 1.718724 \\
Corollary 3.11 & $(3.6)$ & 0.500000 & 0.494565 & 2.000000 & 2.000000 \\
& $(3.7)$ & 0.495918 & 0.497245 & 1.711430 & 1.756447 \\
\hline & $(3.8)$ & 0.193838 & 0.198918 & 4.025371 & 4.398545 \\
Corollary 3.12 & $(3.9)$ & 0.335025 & 0.263240 & 1.961539 & 3.095908 \\
& $(3.10)$ & 0.392906 & 0.316756 & 2.219467 & 3.905806 \\
\hline & $(3.11)$ & 0.500000 & 0.497286 & 1.672098 & 1.704487 \\
Theorem 3.13 & $(3.12)$ & 0.500000 & 0.497286 & 1.672098 & 1.704488 \\
& $(3.13)$ & 0.498578 & 0.497285 & 1.702011 & 1.756271 \\
\hline & $(3.14)$ & 0.495042 & 0.497282 & 1.815545 & 2.012170 \\
Corollary 3.14 & $(3.15)$ & 0.387055 & 0.494565 & 4.433761 & 8.022150 \\
& $(3.16)$ & 0.441725 & 0.497245 & 4.051864 & 7.689797 \\
\hline \multirow{3}{*}{ Corollary 3.15 } & $(3.18)$ & 0.499569 & 0.497285 & 1.680030 & 1.717423 \\
& $(3.19)$ & 0.472919 & 0.496389 & 1.891675 & 1.985557 \\
& $(3.20)$ & 0.498578 & 0.497285 & 1.702011 & 1.756271 \\
\hline Corollary 3.17 & $(3.22)$ & 0.437152 & $\mathbf{0 . 6 4 4 5 5 7}$ & 2.370628 & 1.517975 \\
\hline
\end{tabular}

Table 2: Comparison of bounds given by formulas (3.1)-(3.22). 
Example 3.2. Theorem 3.8 will not give an exclusion region for all octonionic polynomials, but it is not difficult to find one that does. Consider the octonionic polynomial

$$
p_{2}(x)=\boldsymbol{i} x^{6}+\frac{1}{2} x^{5}+(3-4 \boldsymbol{i} \boldsymbol{j}) x^{4}+\frac{\boldsymbol{i} \boldsymbol{k}}{3} x^{3}+(6 \boldsymbol{i}+8 \boldsymbol{j} \boldsymbol{k}) x^{2}+\boldsymbol{i} \boldsymbol{j} \boldsymbol{k} x+1 .
$$

The zeros belong to the following six approximate conjugacy classes

$$
\begin{array}{lll}
{[1.691188+1.861327 \boldsymbol{i}],} & {[-1.690392+1.580116 \boldsymbol{i}],} & {[-0.941026+0.977546 \boldsymbol{i}],} \\
{[0.940187+0.900354 \boldsymbol{i}],} & {[-0.215089+0.226091 \boldsymbol{i}],} & {[0.215131+0.225644 \boldsymbol{i}],}
\end{array}
$$

with 2-norm values approximately equal to $2.5148867,2.313913,1.356881$, $1.301764,0.312058$ and 0.311764 , respectively.

Applying Theorem 3.7 we obtain an upper bound 2.802674 and a lower bound 0.265132 .

Constructing Newton's polygon for this polynomial, we observe that some information from Theorem 3.8 can be obtained only if $k=2$ or $k=4$. Indeed, only $k=2$ gives two positive real roots, 0.392328 and 1.082217 , which means that $p$ has two zeros with norm less than 0.392328 and four with norm greater than 1.082217. Between this gap there are no roots.

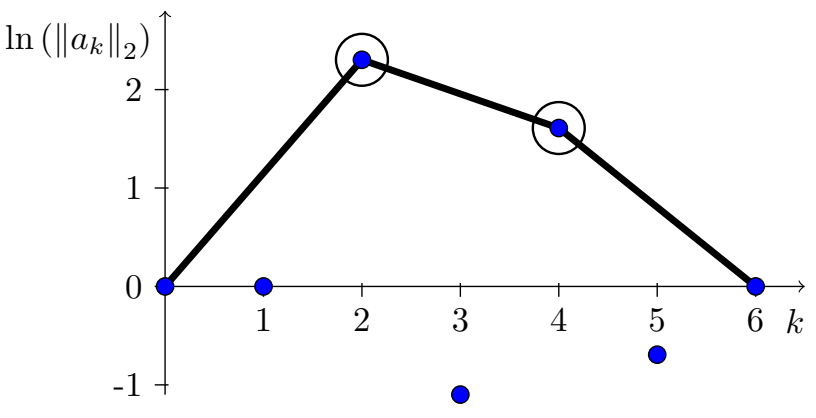

Figure 1: Newton's polygon for polynomial $p_{2}$ indicating the values of $k$ for which it is worth to try to apply Theorem 3.8 .

Example 3.3. In this last example we have generated polynomials of degree $m=3, \ldots, 14$ and for each polynomial we applied Theorem 3.16 with $\kappa=$ $1, \ldots, m-1$. We did this with real polynomials and octonionic polynomials.

In the real case, for each degree $m$ we randomly generated $m$ coefficients, between $10^{-3}$ and $10^{3}$ with random sign, for the monic real polynomial, constructed the respective companion matrix and applied Theorem 3.16 for each $\kappa$, from 1 to $m-1$. The value of $\kappa$ for the greatest upper bound was retained. 
BOUNDS FOR THE ZEROS OF UNILATERAL OCTONIONIC

\begin{tabular}{r|rrrrrrrrrrrr} 
& \multicolumn{10}{c}{ Polynomial's Degree } \\
$\kappa$ & 3 & 4 & 5 & 6 & 7 & 8 & 9 & 10 & 11 & 12 & 13 \\
\hline 1 & $\mathbf{5 3 . 3 9}$ & 5.94 & 10.91 & 8.59 & 7.66 & 6.99 & 6.37 & 5.97 & 5.38 & 5.21 & 4.95 & 4.22 \\
2 & 4.61 & $\mathbf{6 4 . 7 3}$ & 15.19 & 9.70 & 7.43 & 6.41 & 5.21 & 4.37 & 4.06 & 3.63 & 3.03 & 2.84 \\
3 & - & 29.33 & $\mathbf{4 6 . 6 4}$ & 3.43 & 1.18 & 0.92 & 0.93 & 0.83 & 0.69 & 0.81 & 0.60 & 0.52 \\
4 & - & - & 27.26 & $\mathbf{5 4 . 7 0}$ & 3.17 & 0.78 & 0.61 & 0.56 & 0.54 & 0.56 & 0.50 & 0.66 \\
5 & - & - & - & 23.58 & $\mathbf{5 9 . 4 1}$ & 2.62 & 0.48 & 0.47 & 0.34 & 0.43 & 0.38 & 0.28 \\
6 & - & - & - & - & 21.15 & $\mathbf{6 2 . 8 9}$ & 2.44 & 0.40 & 0.32 & 0.38 & 0.27 & 0.23 \\
7 & - & - & - & - & - & 19.39 & $\mathbf{6 6 . 6 6}$ & 2.18 & 0.28 & 0.19 & 0.17 & 0.10 \\
8 & - & - & - & - & - & - & 17.30 & $\mathbf{6 8 . 3 0}$ & 2.00 & 0.23 & 0.16 & 0.13 \\
9 & - & - & - & - & - & - & - & 16.92 & $\mathbf{6 9 . 6 0}$ & 1.88 & 0.10 & 0.15 \\
10 & - & - & - & - & - & - & - & - & 16.79 & $\mathbf{7 1 . 4 8}$ & 1.90 & 0.13 \\
11 & - & - & - & - & - & - & - & - & - & 15.20 & $\mathbf{7 3 . 4 1}$ & 1.97 \\
12 & - & - & - & - & - & - & - & - & - & - & 14.53 & $\mathbf{7 3 . 7 1}$ \\
13 & - & - & - & - & - & - & & - & - & 15.06
\end{tabular}

Table 3: Percentage of cases where the best partition is $\kappa$ for each degree in the real case.

This process was repeated 10000 times for each degree. The result of this experience is presented in Table 3 where the values are given in percentage. This table suggests that, in general, the best partition is the penultimate, and the second best is the last one. Furthermore, as the degree increases the penultimate becomes even more predominant.

In the octonionic case, for each degree $m$ we generated $m$ coefficients for the monic octonionic polynomial. For each coefficient nine random numbers between $10^{-2}$ and $10^{2}$ were generated, eight for the elements of the octonion and one as a factor so that the coefficients had different sizes. The elements of the octonion were also randomly assigned. As in the real case, all values of $\kappa$ were used and the one that gave the best result was retained. This process was repeated 10000 times for each degree. The results are presented in Table 4. In this case, the predominance of the penultimate and last partitions is even greater, having the same tendency as the real case when the polynomials degree increases.

\begin{tabular}{|c|c|c|c|c|c|c|c|c|c|c|c|c|}
\hline \multirow[b]{2}{*}{$\kappa$} & \multicolumn{12}{|c|}{ Polynomial'sDegree } \\
\hline & 3 & 4 & 5 & 6 & 7 & 8 & 9 & 10 & 11 & 12 & 13 & 14 \\
\hline 8 & 27.41 & 0.05 & 0 & 0 & 0 & 0 & 0 & 0 & 0 & 0 & 0 & 0 \\
\hline 16 & 72.59 & 43.22 & 0.45 & 0.06 & 0 & 0 & 0 & 0 & 0 & 0 & 0 & 0 \\
\hline 24 & - & 56.73 & 50.34 & 0 & 0.01 & 0 & 0 & 0 & 0 & 0 & 0 & 0 \\
\hline 33 & - & - & 49.21 & 55.19 & 0 & 0 & 0 & 0 & 0 & 0 & 0 & 0 \\
\hline 40 & - & - & - & 44.75 & 58.10 & 0 & 0 & 0 & 0 & 0 & 0 & 0 \\
\hline 48 & - & - & - & - & 41.89 & 60.89 & 0 & 0 & 0 & 0 & 0 & 0 \\
\hline 56 & - & - & - & - & - & 39.11 & 63.40 & 0 & 0 & 0 & 0 & 0 \\
\hline 64 & - & - & - & - & - & - & 36.60 & 65.12 & 0 & 0 & 0 & 0 \\
\hline 72 & - & - & - & - & - & - & - & 34.88 & 64.62 & 0 & 0 & 0 \\
\hline 80 & - & - & - & - & - & - & - & - & 35.38 & 67.19 & 0 & 0 \\
\hline 88 & - & - & - & - & - & - & - & - & - & 32.81 & 67.57 & 0 \\
\hline 96 & - & - & - & - & - & - & - & - & - & - & 32.43 & 68.51 \\
\hline 104 & - & - & - & - & - & - & - & - & - & - & - & 31.49 \\
\hline
\end{tabular}

Table 4: Percentage of cases where the best partition is $\kappa$ for each degree in the octonionic case. 


\section{Acknowledgements}

R. Serôdio and P. D. Beites were supported by FCT (Fundação para a Ciência e a Tecnologia, Portugal), project UIDB/00212/2020 of CMA-UBI (Centro de Matemática e Aplicações da Universidade da Beira Interior). P. D. Beites was also supported by MINECO (Ministerio de Asuntos Económicos y Transformación Digital, Spain), project MTM2017-83506-C2-2-P. The authors would like to thank Maria Helena Serôdio and Mary Garfield for clarifying some language queries.

\section{References}

[1] Ahmad, S.S., Ali, I.: Bounds for eigenvalues of matrix polynomials over quaternion division algebra. Adv. Appl. Clifford Algebras 26(4), 10951125 (2016).

[2] Barnett, S.: Matrices in control theory with applications to linear programming. Van Nostrand, London (1971).

[3] Beites, P.D., Nicolás, A.P.: An associative triple system of the second kind. Commun. Algebra 44(11), 5027-5043 (2016).

[4] Boukas, A, Fellouris, A.: On octonion polynomial equations. Int. J. Math. Comput. Sci. 1(2), 59-73 (2016).

[5] Cauchy, A.L.: Sur la résolution des équations numériques et sur la théorie de l'élimination. Exercices de Mathématiques. Chez de Bure frères, Paris, 65-128 (1829).

[6] Chapman, A.: Polynomial equations over octonion algebras. J. Algebra Appl. 19(6), 10 pp. (2020).

[7] Datt, B., Govil, N.K.: On the location of the zeros of a polynomial. J. Approx. Theory 24, 78-82 (1978).

[8] Datta, B., Nag, S.: Zero-Sets of quaternionic and octonionic analytic functions with central coefficients. Bull. London Math. Soc. 19(4), 329336 (1987).

[9] Dehmer, M.: On the location of zeros of complex polynomials. Journal of Inequalities in Pure and Applied Mathematics 72(26) (2006).

[10] Dennis, J.E. Jr., Traub, J.F., Weber, R.P.: On the matrix polynomial, lambda-matrix and block eigenvalue problems. Computer Science Department Technical Report, Cornell University, 71-109 (1971).

[11] Deutsch, E.: Matricial norms. Numer. Math. 16, 73-84 (1970). 
[12] Deutsch, E.: Matricial norms and the zeros of polynomials. Linear Algebra Appl. 3, 483-489 (1970).

[13] Eganova, A., Shirokov, M.I.: Orthomatrices and octonions. Rep. Math. Phys. 20, 1-10 (1984).

[14] Eilenberg, S., Niven, I., The fundamental theorem of algebra for quaternions, Bull. Amer. Math. Soc. 50, 244-248 (1944).

[15] Miranda, F., Falcão, M.I.: Modified quaternion Newton methods. In: Murgante B. et al. (eds) Computational Science and Its Applications ICCSA 2014, June 2014. Lecture Notes in Computer Science, vol 8579, 146-161 Springer, Cham.

[16] Falcão, M.I., Miranda, F., Severino, R., Soares, M.J.: Weierstrass method for quaternionic polynomial root-finding. Mathematical Methods in the Applied Sciences 41(1), 423-437 (2018).

[17] Flaut, C., Ştefănescu, M.: Some equations over generalized quaternion and octonion division algebras. Bull. Math. Soc. Sci. Math. Roumanie, 52, 427-439 (2009)

[18] Higham, N.J., Tisseur, F.: Bounds for eigenvalues of matrix polynomials. Linear Algebra Appl. 358, 5-22 (2003).

[19] Horn, R., Johnson, C.: Matrix analysis. Cambridge University, New York (2012).

[20] Jou, Y.-L.: The "Fundamental Theorem of Algebra" for Cayley numbers. Academia Sinica Science Record 3, 29-33 (1950).

[21] Leite, F.S., Vitória, J.: Generalization of the De Moîvre formulas for quaternions and octonions. Estudos de Matemática, Homenagem a Luís de Albuquerque, University of Coimbra, 121-133 (1991).

[22] Marden, M.: Geometry of polynomials. American Mathematical Society, Providence, Rhode Island (1966).

[23] Melman, A.: Generalization and variations of Pellet's theorem for matrix polynomials. Linear Algebra Appl. 439(5), 1550-1567 (2013).

[24] Pellet, A.E.: Sur un mode de séparation des racines des équations et la formule de Lagrange. Bulletin des Sciences Mathématiques et Astronomiques 5(1), 393-395 (1881).

[25] Serôdio, R., Pereira, E., Vitória, J.: Computing the zeros of quaternion polynomials. Comput. Math. with Appl. 42(8-9), 1229-1237 (2001).

[26] Serôdio, R.: On Octonionic polynomials. Adv. Appl. Clifford Algebras $17,245-258$ (2007). 
[27] Serôdio, R.: Construction of octonionic polynomials. Adv. Appl. Clifford Algebras 20, 155-178 (2010).

[28] Serôdio, R., Ferreira, J.A., Vitória, J.: An iterative method to compute the dominant zero of a quaternionic unilateral polynomial. Adv. Appl. Clifford Algebras 28, article 45 (2018).

[29] Serôdio, R., Beites, P.D., Vitória, J.: Eigenvalues of matrices related to the octonions. 4open 2, article 16 (2019).

[30] Singh, G., Shah, W.M.: On the location of zeros of polynomials. Am. J. Comput. Math. 1(1), 1-10 (2011).

[31] Tian, Y.G.: Matrix representations of octonions and their applications. Adv. Appl. Clifford Algebras 10, 61-90 (2000).

[32] Vitória, J.: Matricial norms and lambda-matrices. Revista de Ciências Matemáticas, Universidade Lourenço Marques, 9-28 (1974-75).

[33] Vitória, J.: Matricial norms and the roots of polynomials. Revista de Ciências Matemáticas, Universidade Lourenço Marques, 51-56 (1974-75).

[34] Wilf, H.S.: Perron-Frobenius theory and the zeros of polynomials. Proc. Am. Math. Soc. 12(2), 247-250 (1961).

[35] Ward, J.P.: Quaternions and Cayley numbers. Springer, Dordrecht (1967).

Rogério SERÔDIO,

CMA and Departamento de Matemática,

Universidade da Beira Interior,

Rua Marquês d'Ávila e Bolama, 6201-001 Covilhã, Portugal.

Email: rserodio@ubi.pt

Patrícia BEITES,

CMA and Departamento de Matemática,

Universidade da Beira Interior,

Rua Marquês d'Ávila e Bolama, 6201-001 Covilhã, Portugal.

Email: pbeites@ubi.pt

José VITÓRIA,

University of Coimbra,

Department of Mathematics,

Apartado 3008, EC Santa Cruz, 3001-501 Coimbra, Portugal.

Email: jvitoria@mat.uc.pt 